\title{
Diagnosis and treatment of DVT and prevention of DVT recurrence and the PTS: bridging the gap between DVT and PTS in the primary care setting
} or outpatient ward

\author{
This article was published in the following Dove Press journal: \\ Journal of Vascular Diagnostics and Interventions \\ 24 March 2017 \\ Number of times this article has been viewed
}

\begin{abstract}
Jan Jacques Michiels ${ }^{1-3}$
Wim Moosdorff'

Mildred U Lao'

Hanny Maasland'

Janneke Maria Michiels ${ }^{2,4}$

HA Martino Neumann ${ }^{5}$

Petr Dulicek ${ }^{6}$

Viera Stvrtinova ${ }^{3,7}$

Pavel Poredos ${ }^{3,8}$

Jacques Barth ${ }^{1,3}$

Gualtiero Palareti ${ }^{3,9}$
\end{abstract}

'Primary Care Medicine Medical Diagnostic Center, Rotterdam, ${ }^{2}$ Goodheart Institute, Blood Coagulation and Vascular Medicine Science Center, Rotterdam, the Netherlands; ${ }^{3}$ Central European Vascular Forum, Prague, Czech Republic; ${ }^{4}$ Primary Care Medicine, Leiden University Medical Center, Leiden, ${ }^{5}$ Department of Dermatology, Section Phlebology, Erasmus University Medical Center, Rotterdam, the Netherlands; ${ }^{6} 4$ th Department of Internal Medicine - Hematology, University Hospital in Hradec Kralove and Charles University in Prague, Faculty of Medicine, Hradec Králové, Czech Republic; Interna Medicine Medical Faculty, Comenius University, Bratislava, Slovakia; ${ }^{8}$ University Medical Centre Ljubljana, Department of Vascular Disease, Ljubljana, Slovenia; ${ }^{9}$ Department of Angiology and Blood Coagulation, University Hospital, Bologna, Italy

Correspondence: Jan Jacques Michiels Goodheart Institute, Blood Coagulation and Vascular Medicine Research Center, Erasmus Tower, Veenmos I3, 3069 AT Rotterdam, the Netherlands Email goodheartcenter@upcmail.nl

\begin{abstract}
Duplex ultrasonography (DUS) does pick up alternative diagnoses (AD) including Baker's cyst, muscle hematomas, old deep vein thrombosis (DVT), and superficial vein thrombosis. The sequential use of DUS followed by a sensitive D-dimer test and a clinical score assessment is a safe and effective noninvasive strategy to exclude and diagnose DVT and AD in patients with suspected DVT. DVT patients are recommended to wear medical elastic stockings (MECS) for symptomatic relief of swollen legs during the acute phase of DVT, or when postthrombotic syndrome (PTS) is present. In routine daily practice, discontinuation of anticoagulation at 6 months post-DVT is followed by a subsequent $20 \%-30 \%$ DVT recurrence rate; this is the main cause of PTS after 1-5 years of follow-up. To bridge the gap between DVT and PTS, the frequent occurrence of PTS is best prevented by prolonged anticoagulation, if indicated, based on objective risk factors for DVT recurrence. Post-DVT rapid and complete recanalization on DUS within 1-3 months and no reflux is associated with no development of PTS, obviating the need of MECS; furthermore, anticoagulation can be discontinued after 3-6 months post-DVT. Absence of residual venous thrombosis (complete recanalization) at 3 months post-DVT with no reflux and with a low PTS score is associated with no recurrence of DVT (1.2\% of 100 patient/years). The presence of reflux due to valve destruction, irrespective of the degree of recanalization on DUS at 3-6 months post-DVT, is associated with a high risk of DVT recurrence and symptomatic PTS, indicating the need to wear MECS and extend anticoagulation. Appearance of reflux on DUS at 6 months or 9 months post-DVT in symptomatic PTS patients is associated with increased DVT recurrence in about one-third of post-DVT patients after the discontinuation of anticoagulation. We designed a prospective safety outcome management study to bridge the gap between DVT and PTS, with the aim of reducing the overall DVT recurrence rate to $<3 \%$ patient/years during long-term follow-up.
\end{abstract}

Keywords: deep vein thrombosis, DVT recurrence, postthrombotic syndrome, D-dimer, clinical score

\section{Deep vein thrombosis (DVT) and alternative diagnoses mimicking DVT}

Symptomatic DVT or calf vein thrombosis may be the tip of a "clotted iceberg". The cause of DVT may be idiopathic or provoked, usually in the context of surgery, trauma, pregnancy, and during the postpartum period. Alternative diagnoses (AD) like Baker's cyst, ruptured Baker's cyst, torn plantaris tendon, hematoma, 
muscle tears or pulls, erysipelas, lymphedema, and so on, should also be differentiated from DVT. ${ }^{1}$ Accurate differentiation between AD and DVT, or the absence of both, in outpatients with suspected DVT by a clinical assessment according to the Wells scores is subjective; it depends on experienced supervision by highly specialized clinicians in carefully controlled clinical settings, and it does not appear to be reproducible in multidisciplinary settings in hospitals or in multicenter clinical trials. Consequently, in 1998, we decided to eliminate the "score of minus 2 " for AD. In our prospective DVT management study of more than 2,000 outpatients with suspected DVT, patients were referred to our medical diagnostic center in Rotterdam (the Netherlands) between 2005 and 2012 to exclude or diagnose DVT and AD (Figure 1). ${ }^{2,3}$ In our DVT studies, we used the Rotterdam modification of the Wells clinical score assessment as a fill-in form at the time of DVT and $\mathrm{AD}$ exclusion and diagnosis in the setting of a medical diagnostic laboratory in the primary care setting (which is equivalent to the outpatient hospital setting). ${ }^{2,3}$ The " $\mathrm{AD}$ minus 2" scoring procedure is subjective, not specific, not sensitive, and not reproducible enough in daily clinical hospital and outpatient practice, and it should not be used in daily clinical and outpatient practice. . $^{2,3}$

The advantage of complete duplex ultrasonography (CDUS) of the calf, popliteal, femoral, and iliac veins is that it does pick up distal and proximal DVT and AD, such as Baker's cysts, muscle hematomas, old DVTs, and superficial vein thrombosis (SVT). AD with a negative duplex ultrasonography (DUS) include leg edema, varices, erysipelas, and so on. The NPV of CDUS by experienced specialists was $99.5 \%$ in the medical diagnostic laboratory setting or outpatient ward in three large management studies with a prevalence of DVT from $14 \%-33 \% .{ }^{4-6}$ CDUS in a prospective clinical study of 623 patients with suspected DVT detected AD in a total of 248 cases $(60.5 \%) .{ }^{4}$ An underlying disease and abnormal CDUS was found in 172 $(42 \%)$ cases, with venous reflux in 93 , muscular lesions in 20, hematoma in 24, and Baker's cyst in 35 (eight ruptured). In addition, an underlying disease and normal DUS was found in 77 (18.5\%) cases, including peripheral artery disease, cutaneous infection, neurologic/rheumatologic disease, and lymphedema. These clinical observations indicate that CDUS is far superior to phlebography and should become

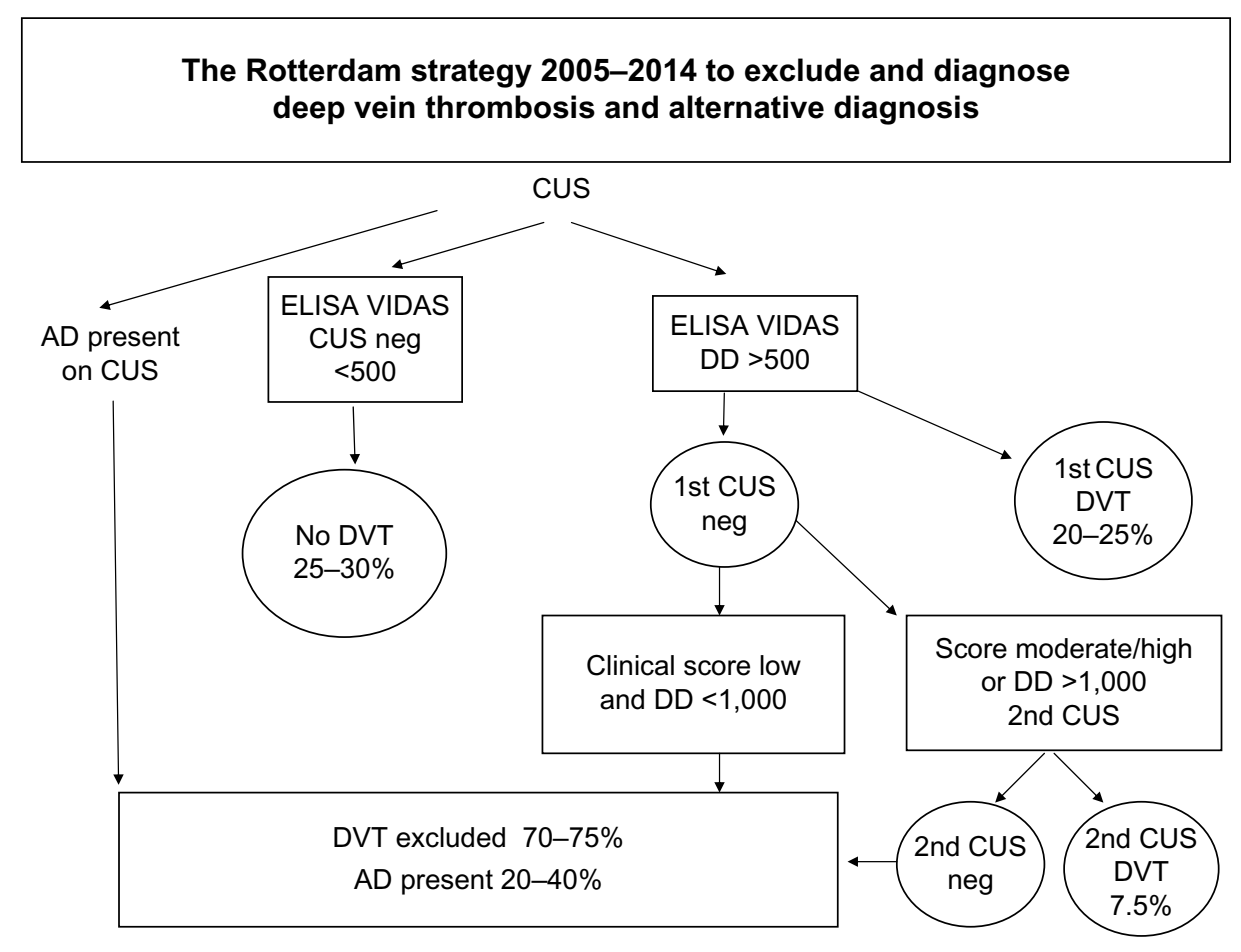

Figure I The Rotterdam strategy to safely exclude and diagnose DVT and AD with a sensitivity and specificity near I00\% by the sequential use of CUS, DD, and clinical score assessment.

Note: $\operatorname{VIDAS}^{\circledR}$ (BioMerieux, Lyon, France).

Abbreviations: CUS, compression ultrasonography; ELISA, enzyme-linked immunosorbent assay; DD, D-dimer; neg, negative; DVT, deep vein thrombosis; AD, alternative diagnosis. 
the first objective step for the diagnosis and exclusion of DVT and AD.

\section{Sequential DUS, D-dimer, and clinical score}

Based on personal experiences and evidence-based objective diagnostic tools, in 2005 we developed the Rotterdam concept (Figure 1) to safely exclude and diagnose DVT, 2,3,7-10 as per the following reasoning. First, a negative CDUS and a negative enzyme-linked immunosorbent assay (ELISA) D-dimer $\left(\right.$ VIDAS $^{\circledR}[$ BioMerieux, Lyon, France $]<500 \mathrm{ng} / \mathrm{mL}$ ) test excluded DVT with a sensitivity and specificity near $100 \%$, irrespective of the clinical score assessment. After a first negative CDUS, the prevalence of DVT in routine daily practice is uniformly low at $2 \%-3 \% .^{7-10}$ The combination of a negative qualitative D-dimer test (SimpliRED or Simplify) and low clinical score should be estimated as not being safe enough, mainly because the prevalence of DVT in the low clinical score group may vary widely $(3 \%-12 \%){ }^{2,3}$ The safest, most effective, and sensitive approach to determine DVT and AD exclusion is to start with objective testing via CDUS, followed by a sensitive D-dimer test without the use of or need for clinical score assessments (Figure 1). A first negative CDUS and a negative sensitive D-dimer test (ELISA VIDAS $<500 \mathrm{ug} / \mathrm{mL}$ ) excludes DVT, irrespective of the clinical score assessment (left arm; Figure 1). The combination of a first negative compression ultrasonography (CUS), a low clinical score with a VIDAS D-dimer level of $<1,000 \mathrm{ug} / \mathrm{mL}$, a Tina-quant ${ }^{\circledR}$ (Hoffman-La Roche Ltd, Basel, Switzerland) level $<800 \mathrm{ug} / \mathrm{mL}$, or a negative SimpliRED or Simplify test safely excluded DVT with an NPV of near to $100 \%$ in four prospective outcome studies $^{7-10}$ (middle arm; Figure 1). Those patients with a clear suspicion of DVT with a moderate to high Rotterdam clinical score and with pronounced increased D-dimer levels (VIDAS $>1,000 \mathrm{ug} / \mathrm{mL}$; Tina-quant $>800 \mathrm{ug} / \mathrm{mL}$ ) or a positive qualitative D-dimer SimpliRED or Simplify test are candidates for a repeated DUS of the legs after 1 week (right arm; Figure 1). ${ }^{3}$ The Rotterdam concept in Figure 1 is under investigation in two prospective management studies of more than 2,000 patients, which were conducted in the period between 2006-2012 (Michiels et al unpublished data 2014).

\section{DVT recurrence related to normal or abnormal CUS at 3 months post-DVT}

In a study by Siragusa et al, ${ }^{11}$ residual vein thrombosis (RVT) was detected in $180(69.8 \%)$ of 258 DVT patients at 3 months
post-DVT. In the cohort of 78 patients who underwent rapid recanalization within 90 days (3 months), anticoagulation was discontinued at 3 months post-DVT; the risk of recurrent venous thromboembolism (VTE) during follow-up was $1.3 \%$ (95\% confidence interval $[\mathrm{CI}], 1 \%-7 \%$; Table 1 ). The proportion of provoked versus unprovoked DVT was $64 \%$ and $36 \%$, respectively. The group of 180 DVT patients with RVT (with no or partial recanalization on DUS) were randomized to stop anticoagulation at 3 months post-DVT (number $=92$ ) or to continue the anticoagulant for 9 additional months, while discontinuing it 12 months post-DVT. During follow-up, from the time that the anticoagulant was discontinued, recurrent VTE events occurred in 25 of 92 (15\%) with RVO after discontinuance of anticoagulation at 3 months post-DVT and in 17 of $88=19 \%$ after discontinuance of anticoagulation at 12 months post-DVT (Figure 2). Interestingly, the proportion of provoked versus unprovoked DVT in patients with residual vein occlusion (RVO) (incomplete recanalization) at 3 months post-DVT was $23 \%$ versus $77 \%$, respectively, again indicating that this distinction is artificial in terms of its risk on DVT recurrence. ${ }^{10}$

In the extended DACUS study 409 patients with a first unprovoked DVT were evaluated for the presence of residual vein thrombosis (RV)T at 3 months post-DVT. DVT patients without RVT suspended VKA after 3 month, while those with RVT continued oral anticoagulation for up to 2 years 11 .

Table I DVT recurrence is related to normal CUS at 3 months post-DVT (rapid recanalization) versus abnormal CUS at 3 months post-DVT (delayed recanalization) in the DACUS and extended DACUS Studies ${ }^{10,11}$

\begin{tabular}{|c|c|c|c|}
\hline $\begin{array}{l}\text { DACUS study CUS } \\
\text { at } 3 \text { months } \\
\text { post DVT }\end{array}$ & $\begin{array}{l}\text { CUS Normal no } \\
\text { RVT } \\
\text { N (\%) }\end{array}$ & $\begin{array}{l}\text { CUS Abnormal } \\
\text { RVT } \\
\text { N (\%) }\end{array}$ & $P$-value \\
\hline Number of patients & 78 & 180 & \\
\hline Provoked DVT & $50(64 \%)$ & 44 (24\%) & \\
\hline Idiopathic DVT & $28(36 \%)$ & 138 (76\%) & \\
\hline $\begin{array}{l}\text { DVT recurrence after } \\
\text { discontinuation of VKA } \\
\text { ( } 3 \text { year follow-up) }\end{array}$ & I (I.3\%) & $42(23 \%)$ & \\
\hline $\begin{array}{l}\text { Extended DACUS } \\
\text { study CUS at } \\
3 \text { months post-DVT } \\
\end{array}$ & No RVT & RVT & \\
\hline Number of patients & 136 & 258 & \\
\hline $\begin{array}{l}\text { VTE recurrence after } \\
\text { VKA discontinuation }\end{array}$ & $2(1.4 \%)$ & 27 (10.4\%) & 0.0026 \\
\hline $\begin{array}{l}\text { VTE recurrences \% per } \\
100 \text { person-years }\end{array}$ & $1.4 \%$ & $10.4 \%$ & 0.022 \\
\hline
\end{tabular}

Abbreviations: RVT, residual vein thrombosis; DACUS, Duration of Antiocoagulation based on Compression Ultrasonography Study; VKA: vitamin K antagonist; VTE, venous thromboembolism; CUS, compression ultrasonography; DVT, deep vein thrombosis. 


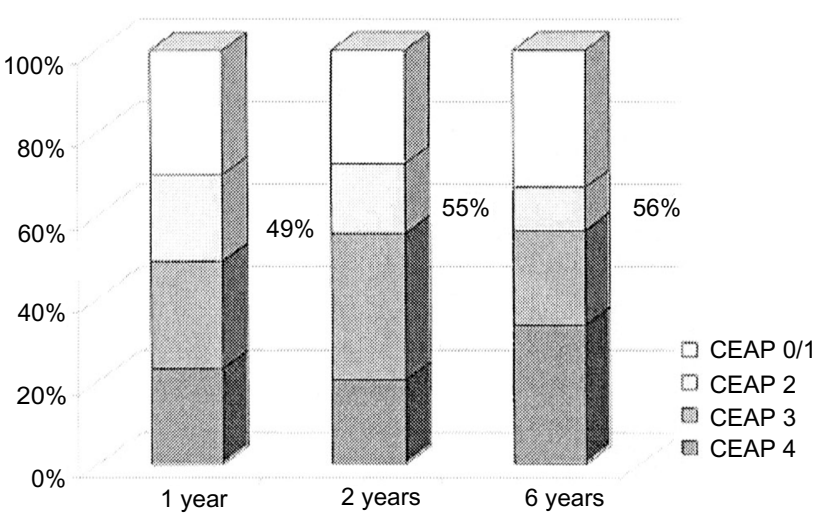

Figure 2 Incidence of PTS according to the CEAP classification in a well controlled study of 93 DVT patients during long-term follow-up.

Notes: Courtesy of Dr Roumen-Kappe, Dutch DVT-PTS Study Group.

Abbreviations: CEAP, Clinical-Etiology-Anatomic-Pathophysiological; PTS, postthrombotic syndrome; DVT, deep vein thrombosis.

Among 409 patients evaluated for unprovoked DVT, 33.2\% (136 of 409 patients) did not have RVT and VKA was stopped after 3 months. The remaining 273 (66.8\%) patients with RVT received anticoagulants for an additional 21 months; during this period of treatment, recurrent venous thromboembolism and major bleeding occurred in $4.7 \%$ and $1.1 \%$ of patients, respectively. However, after VKA suspension, the rates of recurrent thrombotic events were $1.4 \%$ the no-RVT group and $10.4 \%$ in the RVT group (relative risk $=7.4 ; 95 \%$ confidence interval $=4.9-9.9)$. These results indicate that in patients without RVT, a short period of treatment with a VKA is sufficient; in those with persistent RVT, treatment extended to 2 years substantially reduces, but does not eliminate, the risk of recurrent DVT.

\section{The postthrombotic syndrome (PTS)}

The Clinical-Etiology-Anatomic-Pathophysiological (CEAP) classification for PTS is well known and widely applied by phlebologists. The simplified CEAP classification for PTS is not used by clinicians or vascular medicine specialists (Table 2). In 1992, Prandoni developed an original standardized scale for the assessment of PTS in post-DVT legs (Paolo Prandoni, Institute of Medical Semeiotics, University Hospital of Padua, Italy; personal communication). Each subjective sign and objective symptom was scored as $0,1,2$, or 3 based on clinical judgment. ${ }^{12,13}$ The five signs and seven symptoms of the original Prandoni score are taken over by PTS investigators as the Villalta in the literature. ${ }^{14}$ The Prandoni subjective signs and objective symptoms (Villalta score) have never been evaluated against the objective CEAP scoring system (Tables 2 and 3). It is mandatory that the Prandoni (Villalta) scoring system of the subjective signs and objective symptoms of early PTS be verified against the objective CEAP scoring system in
Table 2 Simplified clinical part of the CEAP classification for the severity of chronic venous insufficiency (either primary or secondary) ${ }^{12}$

\begin{tabular}{ll}
\hline Classification & Symptom \\
\hline Clinical: C & \\
C0 asymptomatic & No visible varicose veins \\
Cl & Spider or reticular veins \\
C2 & Varicose veins \\
C3 & Edema \\
C4a & Pigmentation or eczema \\
C4b & Lipodermatosclerosis or atrophie blanche \\
C5 & Skin changes with healed ulceration \\
C6 & Skin changes with active ulceration \\
\hline
\end{tabular}

Abbreviations: CEAP, Clinical-Etiology-Anatomic-Pathophysiological; C, clinical.

Tables 2 and 3 at 3 months, 6 months, and 12 months postDVT up to $2-5$ years post-DVT in prospective management studies. This statement is of particular interest, since the data from the literature are convincing in showing that PTS is present in about $50 \%$ of post-DVT patients at 1 year postDVT using the Villalta score and are not compared with the CEAP score. ${ }^{15,16}$

\section{DVT recurrence and $\mathrm{PTS}$}

In a post hoc analysis of a selected large group of 452 patients with unprovoked DVT (Table 2), Le Gal et al ${ }^{17}$ performed

Table 3 Interpretation of the clinical scoring system according to Prandoni for the assessment of early PTS in the early period of 3-12 months post-DVT, up to 2 years post-DVT ${ }^{13,14}$

\begin{tabular}{lll}
\hline $\begin{array}{l}\text { Five subjective } \\
\text { symp: identical } \\
\text { to symp of CEAP }\end{array}$ & Seven objective signs & $\begin{array}{l}\text { All belong } \\
\text { to C of } \\
\text { CEAP } 16\end{array}$ \\
\hline $\begin{array}{l}\text { Heaviness of the foot/leg } \\
\text { Pain in the calf/thigh }\end{array}$ & $\begin{array}{l}\text { Pretibial edema } \\
\text { Induration of the skin }\end{array}$ & $\mathrm{C} 3$ \\
$\begin{array}{l}\text { Cramps in the calf/thigh } \\
\text { Pruritis }\end{array}$ & $\begin{array}{l}\text { Hyperpigmentation } \\
\text { Paresthesia }\end{array}$ & $\mathrm{C} 4$ \\
& $\begin{array}{l}\text { Redness } \\
\text { Pain during calf }\end{array}$ \\
& compression (post-DVT) & \\
& Ulceration of the skin & $\mathrm{C} 5, \mathrm{C} 6$ \\
\hline
\end{tabular}

Notes: Each sign or symptom is graded with a score of I, 2, or 3 ( $\mathrm{I}=$ mild; $2=$ moderate; $3=$ severe). Ulceration of the skin is 4 points. The presence of a leg ulcer is a late complication of PTS, consistent with severe CVI (CEAP 5, CEAP 6). Definition of clinical PTS according to Prandoni (Villalta): absent, score $\leq 5$; mild to moderate, score between 5-10 and 10-15, respectively, at two consecutive visits; severe, score $\geq 15$ on two consecutive occasions. Conclusion: the Prandoni or Villalta scoring system for PTS is a combination of subjective signs and complaints of PTS and evaluation of objective leg swelling and skin lesions according to the CEAP classification by an internist without expert dermatological evaluation and objective testing with CUS. Moreover, this scoring system should be used at I month, 3 months, 6 months, 9 months, and I year post-DVT for the assessment of the disappearance or persistence of DVT symptoms and the appearance of mild, moderate, and severe PTS symptoms.

Abbreviations: PTS, postthrombotic syndrome; DVT, deep vein thrombosis; CEAP, Clinical-Etiology-Anatomic-Pathophysiological; C, clinical; CVI, chronic venous insufficiency; CUS, compression ultrasonography; symp, symptoms. 
DUS at 6 months if the post-DVT patient had signs or symptoms 5-7 months post-DVT, when the oral anticoagulation (OAT) was stopped. In the study by LE Gal et al, ${ }^{17}$ overt PTS was present in 184 of 452 (46\%) cases, with symptoms of hyperpigmentation (CEAP $\geq 4$ ), edema (CEAP $>3$ ), or redness in either leg (Table 2). Recanalization on DUS was complete in 220 patients and abnormal in 231 patients, with minimal wall thickening in eleven, partial thrombus resolution in 78, minimal thrombus resolution (obstruction) in 23 , and missing data in 67, but reflux was not measured. During the follow-up period (once patients were off the OAT), 45 of 231 patients with abnormal CUS (19.5\%) and 32 out of 220 patients $(14.6 \%)$ with normal DUS (complete recanalization) had recurrent VTE. The conclusion is that the degree of recanalization (complete versus incomplete) alone at 6 months post-DVT is not predictive of DVT recurrence and the presence or development of PTS.

Rodger et al and Verhovsek et al ${ }^{18-20}$ conducted a multicenter, prospective cohort clinical decision rule derivation of 646 patients with a first unprovoked VTE treated with OAT for about 6 months; patients were followed for recurrent VTE after OAT discontinuation. PTS signs, as assessed by the presence of hyperpigmentation, edema, and redness (HER), appeared to make biological and clinical sense as important risk factors for DVT recurrence and the onset of embolic complications. The presence of HER as can be quantified by the Villalta scoring system (defined by Prandoni), as used by clinicians, and/or by objective CEAP criteria used by phlebologists - even at normal dimer levels - after the discontinuation of OAT, irrespective of CUS abnormalities at 6 months to 12 months post-DVT are predicted to become the strongest predictors of DVT recurrence as the cause of PTS development or progression.

\section{Clinical features of PTS}

A Dutch study ${ }^{21}$ conducted in 2004 prospectively evaluated the incidence and severity of PTS in 93 DVT patients under careful clinical surveillance using the CEAP classification (Figure 2). This study confirmed the findings from previous studies, whereby only half of DVT patients had no clear evidence of PTS (CEAP 0/1 and CEAP 2; Figure 2). In this study, the cumulative incidence of PTS (CEAP 3 and CEAP 4 ) increased from $49 \%$ after 1 year to $55 \%$ and $56 \%$ after 2 years and 6 years, respectively. Class C5 (active ulceration) and C6 (healed ulcers) did not occur while on long-term treatment with medical elastic stockings (MECS) (Figure 2). ${ }^{21}$

Aschwanden et $\mathrm{al}^{22}$ studied the effect of prolonged treatment with MECS to prevent PTS in a randomized clinical trial (patients were recruited between 1997 and 2004 comparing intervention with no intervention [control] in case of mild PTS [CEAP 1 and 2]). In this study, DVT patients completed the recommended anticoagulation treatment with low molecular weight heparin followed by a vitamin $\mathrm{K}$ agonist (VKA) for 6 months. The inclusion criteria at 6 months post-DVT were early PTS with CEAP C0/1, CEAP C2, or CEAP C3 without skin lesions, and the exclusion criteria were CEAP C4-C6 with skin lesions. The primary end point was defined as a progression of PTS (CEAP C1-C3) without skin lesions to PTS with skin changes (CEAP C4 or higher). The intention-to-treat population consisted of 169 patients. The incidence and distribution of the post-thrombotic syndrome (PTS) according to the CEAP classification (Table 3) during long-term follow-up. The completeness of follow-up was $85 \%$ in the intervention group and $75 \%$ in the control group, with a mean follow-up of 3.2 years in the intervention group and 2.9 years in the control group. The secondary end point was independently evaluated and defined by the presence of five PTS-associated symptoms, according to Widmer: pain; heaviness; sensation of heat; tension; and tiredness of the affected limb. Overall, the primary end point (CEAP $\geq 4$ or higher; skin lesions) developed in $13 \%$ of the intervention group and $20 \%$ of the control group. The secondary end point of at least one of the five Widmer symptoms was recorded in $12.2 \%$ of follow-up visits in the intervention group and $16.5 \%$ of follow-up visits in the control group. Recurrent DVT and female sex were major risk factors for the development of PTS $(\geq \mathrm{C} 4$ or higher; skin lesions) after 2-6 years' follow-up in patients with reflux and/or obstruction on DUS at the time of inclusion, at 6 months post-DVT. ${ }^{22}$

\section{Pathophysiology and objective monitoring of PTS}

Meissner et $\mathrm{al}^{23}$ studied the relationship between time to complete recanalization post-DVT (lysis time of the leg vein clot) and the development of reflux in patients with a first episode of DVT at 3-month intervals during the first year (Figure 3). Duplex criteria for complete occlusion were defined as the absence of detectable flow, either spontaneous or with augmentation, in an incompressible venous segment. Partial occlusion was defined as normal or diminished flow, either spontaneous or with augmentation, in an incompletely compressible venous segment. Complete lysis of the leg vein clot (recanalization) was presumed to have occurred when spontaneous phasic flow returned and the vein was completely compressible. ${ }^{23}$ For the posterior tibial veins, flow detected after distal augmentation in a completely compressible vein is accepted as evidence of complete recanalization (lysis of 


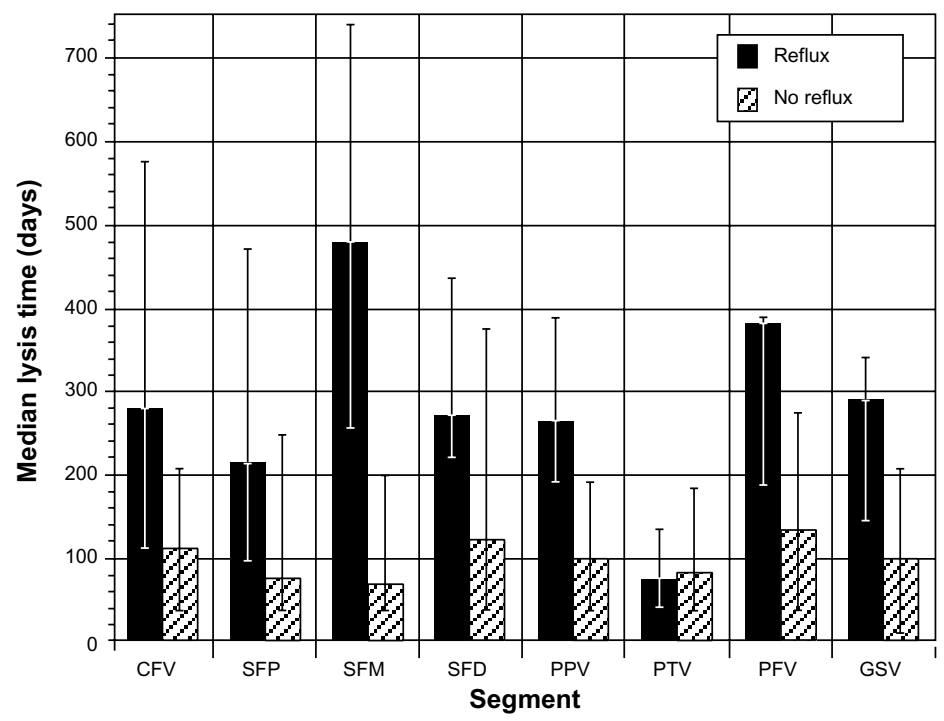

Figure 3 Rapid recanalization of thrombosed legs in DVT patients.

Notes: A rapid recanalization of thrombosed legs in DVT patients within 3 months (median thrombosis lysis time from DVT to complete recanalization in I00 days; striped bars) in 118 post-DVT patients was associated with no reflux in all segments (striped bars). These DVT patients with rapid recanalization had popliteal or distal DVT in 68 of 118 and proximal femoral DVT in 46 of II8. A delayed recanalization of thrombosed legs in DVT patients (median thrombosis lysis time from DVT to the complete recanalization of all vein segments of $>6$ months to about 9-12 months [black bars]) in 65 DVT patients was associated with the development of reflux as the main determinant of symptomatic PTS (black bars). The majority of DVT patients with delayed recanalization and reflux had proximal femoral DVT in 42 of 52 patients and popliteal DVT in 10 of 52 DVT patients. ${ }^{23}$

Abbreviations: CFV, common femoral vein; SFP, superficial femoral vein; SFM, middle superficial femoral vein; SFD, distal superficial vein; PPV, popliteal vein; PTV, posterior tibial vein; PFV, proximal femoral vein; GSV, greater saphena vein; DVT, deep vein thrombosis; PTS, postthrombotic syndrome.

the leg vein clot). The DVT-Lysis-Reflux Study ${ }^{23}$ in Figure 3 shows that a rapid and short leg clot lysis time (in terms of complete recanalization of the DVT within 1-3 months; lysis time 65-130 days) is associated with no development of reflux; patients usually have no PTS, and they do not need to continue the use of MECS 3 months post-DVT. This accounts for about two-thirds of patients with a first event of DVT. The DVT patients with rapid recanalization (lysis time) within 3 months post-DVT had calf or popliteal vein thrombosis at diagnosis in 68 of $118(58 \%)$, and proximal femoral vein thrombosis in 46 of 118 (40\%) DVT patients. The majority of DVT patients with delayed recanalization and reflux had proximal femoral DVT (42 of 52 patients) and popliteal DVT (ten of 52 patients). If the time to recanalization was delayed (lysis time: 214-375 days) from more than 3 months to 6-9 or 12 months then those DVT patients developed reflux as a main determinant of the venous system (popliteal, superficial, and common femoral vein [figure for the development of PTS]; Figure 3). ${ }^{23}$

As shown in Figure 4, reflux due to a loss of valve competence leading to ambulatory venous hypertension and diversion of venous flow through incompetent perforator veins appear to play an important role in the development of the late complications of PTS. ${ }^{24}$ In this study of 123 legs with DVT (107 patients), about two-thirds of the involved legs had developed valve incompetence. ${ }^{24}$ The distribution of reflux at the end of the first year follow-up study was the following: popliteal vein, $58 \%$; femoral vein, $37 \%$; greater saphenous vein, $25 \%$; and posterior tibial vein, $18 \%$. Reflux appeared

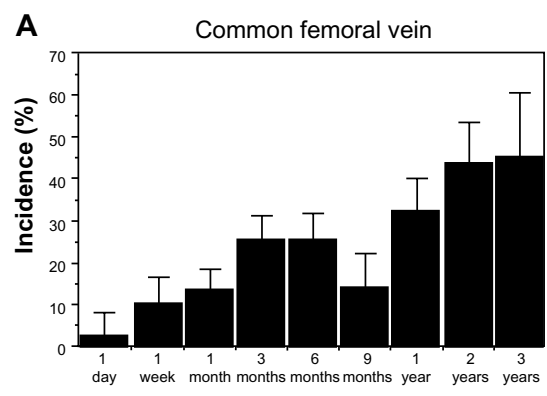
Popliteal vein

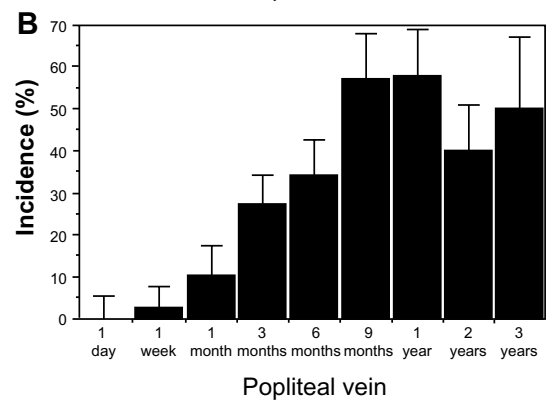

Figure $4(A, B)$ Localization and incidence of reflux in deep veins in post-DVT patients who developed reflux on DUS at the different follow-up intervals post-DVT. Notes: Follow-up intervals were as follows: I day; I week; I month; 3 months; 6 months; 9 months; I year; 2 years; and 3 years in the 1993 landmark Leg Thrombosis Lysis-Reflux study. Courtesy of Dr Markel, International Union of Angiology: IUA.

Abbreviations: DVT, deep vein thrombosis; DUS, duplex ultrasonography. 

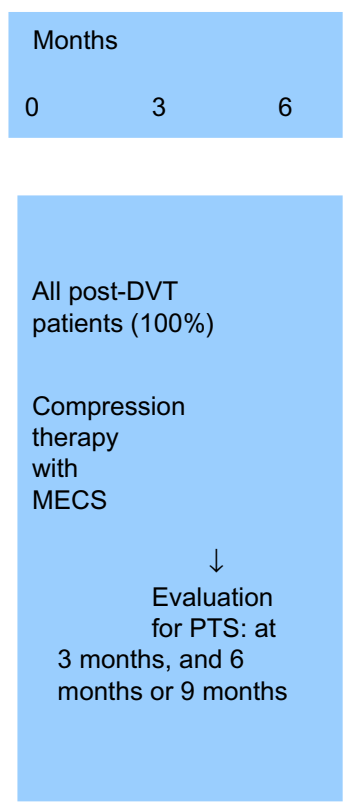

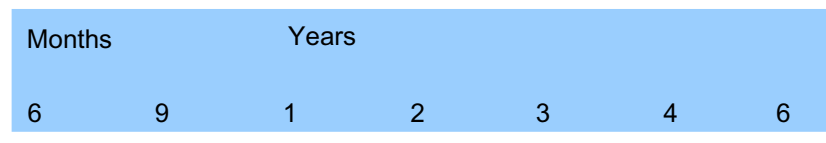

Study arm 1: no PTS

Recanalization and no reflux: no stockings

Study arm 2: reflux, venous pressure normal

No PTS: MECS versus no MECS

\section{Study arm 3: PTS \\ Reflux, increased venous pressure $\rightarrow$ MECS}

\section{Study arm 4: PTS}

Obstruction $=$ No recanalization

Reflux, no reflux $\rightarrow$ MECS

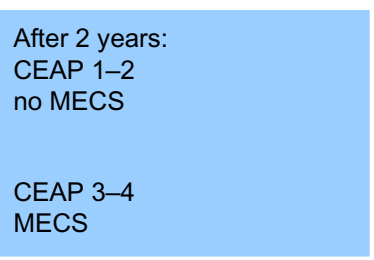

Figure 5 The 2007 study design by Neumann and Michiels to bridge the gap between DVT and PTS, as they relate to the indication of wearing MECS: therapeutic implications. Notes: Study arm I: Rapid recanalization, no reflux, and no PTS at 3 months post-DVT obviates the need to wear stockings and be treated with anticoagulation at 6 months post-DVT. Study arms 3 and 4: Delayed recanalization and/or, reflux on DUS and very early signs of PTS dictates the need for extended (even lifelong) anticoagulation to prevent DVT recurrence as the cause of PTS progression. Study arm 2: Complete recanalization, but reflux on DUS and no PTS (CEAP 0), which may indicate that compensated reflux has a normal ambulatory venous pressure due to an intact calf vein pump (the 2007 Neumann concept, as interpreted by Michiels et al ${ }^{47-49}$ ).

Abbreviations: DVT, deep vein thrombosis; MECS, medical elastic stockings; PTS, postthrombotic syndrome; CEAP, Clinical-Etiology-Anatomic-Pathophysiological; DUS, duplex ultrasonography.

with obstruction and/or reflux on DUS, it was clearly shown that MECS will not prevent DVT recurrence 6 months post-DVT, and that the effect of MECS on PTS symptom reduction are observed after a long-term follow-up of more than 5 years in females but not in males.

\section{The European approaches to DVT and PTS}

Objective measurement of PTS according to CEAP - and of reflux and the degree of recanalization on DUS - at 3 months and 6 months post-DVT is mandatory in evaluating the main

\begin{tabular}{l} 
Months (M) DUS \\
$\begin{array}{l}\text { All post-DVT } \\
\text { patients (100\%) } \\
\text { Compression } \\
\text { therapy } \\
\text { with medical elastic } \\
\text { compression } \\
\text { stockings: MECS } \\
\quad \text { Evaluation } \\
\text { for PTS: Prandoni } \\
\text { score at } 1 \text { month, } \\
3 \text { months, } 6 \text { months, } \\
9 \text { months and } 12 \text { months }\end{array}$ \\
\hline
\end{tabular}

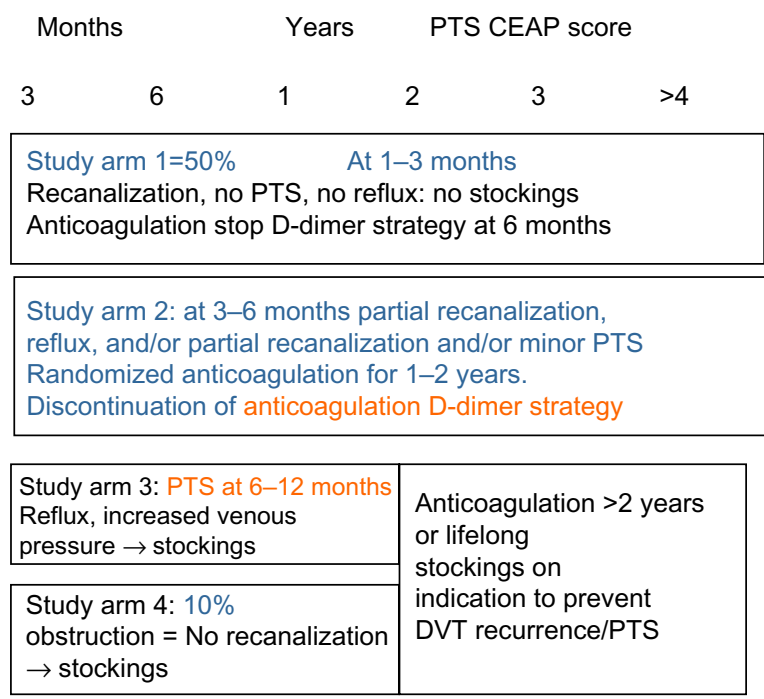

Figure 6 Extension of the Neumann-Michiels ${ }^{49}$ study design to bridge the gap between DVT and PTS by the compelling and objective need to extend the duration of anticoagulant treatment, preferentially with low-dose NOAC (Rivaroxaban ${ }^{\circledR} 10 \mathrm{mg} /$ day; Apixaban ${ }^{\circledR} 2.5 \mathrm{mg} /$ day) in post-DVT patients, according to an objective risk assessment for DVT risk recurrence.

Notes: At the time that the termination of regular anticoagulant treatment is or may be indicated, the discontinuation of oral anticoagulation should follow the D-dimer strategy of the PROLONG and DULCIS studies. ${ }^{33,51}$ Rivaroxaban (Bayer, Leverkussen, Germany); Apixaban (Pfizer, New York City, NY, USA).

Abbreviations: DVT, deep vein thrombosis; MECS, medical elastic stockings; PTS, postthrombotic syndrome; NOAC, novel oral anticoagulants; CEAP, Clinical-EtiologyAnatomic-Pathophysiological. 
Table 5 Outcome of DVT patients with a first IDVT and in patients with a first EDVT

\begin{tabular}{lllllll}
\hline N of limbs & CEAP 0-I & CEAP 2-3 & CEAP 4 & CEAP 5-6 & Reflux/obstruction & Recurring DVT \\
\hline 4 I IDVT & 15 & 20 & 6 & 0 & 15 & 5 \\
$\begin{array}{l}\text { I I symptomatic } \\
79 \text { EDVT }\end{array}$ & 10 & 40 & 21 & 8 & 61 & 16 \\
7 I symptomatic & 10 & & & \\
\hline
\end{tabular}

Note: Symptomatic = symptomatic during a median follow-up of 3.4 years, indicating that half of IDVT and the majority of EDVT limbs become symptomatic for PTS according to the CEAP classification. Data from Labropoulos et al. ${ }^{27}$

Abbreviations: DVT, deep vein thrombosis; IDVT, isolated deep vein thrombosis; EDVT, extended deep vein thrombosis; CEAP, Clinical-Etiology-AnatomicPathophysiological; PTS, postthrombotic syndrome.

determinants for PTS and DVT recurrence in a prospective safety outcome study, according to the proposed study designs shown in Figures 5-7. The rationale is based on the demonstration of a relationship between persistent RVT and the risk of VTE recurrence in two prospective studies. ${ }^{11,29} \mathrm{In}$ one study, ${ }_{11}^{11}$ RVT at 3 months post-DVT was absent in $30 \%$ of post-DVT patients, which was associated with a very low recurrence rate of DVT of $1.2 \%$ during a 2-year follow-up period (score $=0$ at 3 months; Table 4 ). The presence of RVT at 3 months post-DVT in two-thirds of post-DVT patients was associated with a high DVT recurrence rate of $27 \%$ during a 2-year follow-up period after the discontinuation of regular anticoagulation (score $\geq 3$ or more; Table 4 ). The prospective Prandoni et a ${ }^{29}$ study of 313 consecutive DVT patients RVT at any time post-DVT is a risk factor for recurrent VTE. In this study, repeated DUS of the common femoral and popliteal veins was performed at 3 months, 6 months, 12 months, 24 months, and 36 months post-DVT. The cumulative incidence of normal DUS (no RVT) was 39\%, 58\%, 69\%, and $74 \%$ at 6 months, 12 months, 24 months, and 36 months post-DVT, respectively. Of 58 recurrent episodes of VTE, 41 occurred at the time of RVT. The hazard ratio for recurrent VTE was 2.4 for persistent RVT, as compared to complete vein recanalization. ${ }^{29}$

Palareti et al, ${ }^{30,31,33}$ as well as subsequent studies, showed that DVT recurrence of about $5 \%$ per 100 patient year with normal D-dimer levels and 10\%-15\% per 100 patient years with increased D-dimer levels 1 month after discontinuation of anticoagulation. This difference was independent from other factors like thrombophilia or the presence or absence of RVT. Post-DVT patients with increased D-dimer levels after the discontinuation of anticoagulation belonged to the group of symptomatic post-DVT patients at high risk for developing PTS (score $\geq 3$; Table 4). In the PROLONG study, ${ }^{30}$ extended anticoagulation in post-DVT patients with increased D-dimer levels reduced the risk of DVT recurrence from $11 \%$ patient/years to $<2 \%$ per 100 patient/years. In post-DVT patients with a normal D-dimer level, on the month after the discontinuation of regular anticoagulation, the incidence of
DVT recurrence still increased to $4.4 \%$ patient/years. This implies that the segregation of low risk versus high risk of DVT recurrence by D-dimer levels alone in the PROLONG ${ }^{30}$ setting is specific but not sensitive enough. This may imply that DVT recurrence in those patients with either a normal or increased D-dimer very likely does occur in those with incomplete RVT and in complete RVT after 6 months with reflux score 3 or more (Table 4). This important observation has been confirmed by Latella et $\mathrm{al}^{34}$ in their prospective study of 305 DVT patients selected for quantitative ELISA D-dimer (VIDAS) measurements 4 months post-DVT. Of these 305 post-DVT patients, $46 \%$ developed PTS (according to the Villalta score); $25 \%$ developed mild PTS, $13 \%$ developed moderate PTS, 7\% developed severe PTS, and $54 \%$ did not develop PTS during the 24-month follow-up period. Mean D-dimer levels measured 4 months post-DVT were significantly higher in patients with PTS versus those without PTS $(712 \mu \mathrm{g} / \mathrm{L}$ versus $444 \mu \mathrm{g} / \mathrm{L} ; P=0.02)$. At the time of D-dimer level measurement, 213 patients were treated with anticoagulants. Venous reflux assessment at 12 months in 116 patients moderate to severe PTS was present in 51\%. Reflux was detected in $65 \%$ of patients with PTS compared to $43 \%$ of those without PTS, indicating that reflux on DUS is a minor relative risk, but it is not the only determinative factor, for PTS development.

The Maastricht study evaluated 125 consecutive DVT patients with confirmed proximal DVT ( $22 \%$ had a history of previous VTE), who were followed for 2 years using the Villalta clinical scores on four consecutive visits: 3 months; 6 months; 12 months; and 24 months post-DVT. ${ }^{35}$ After 6 months, patients with Villalta scores $\leq 4$ in the absence of reflux on DUS were allowed to discontinue MECS therapy. If reflux was present, two consecutive scores $\leq 4$ were needed to discontinue MECS therapy (asymptomatic compensated reflux, study arm 2; Figure 5). In the Maastricht study, MECS therapy was discontinued in $17 \%$ of patients at 6 months, in $48 \%$ at 12 months, and in $50 \%$ at 24 months. ${ }^{35}$ These observations are in line with those of the Brandjes et al, ${ }^{25}$ Prandoni et al, ${ }^{26}$ and Kahn et $\mathrm{al}^{28}$ studies, obviating the need for MECS in 
DVT PTS bridging the gap 2014/2015

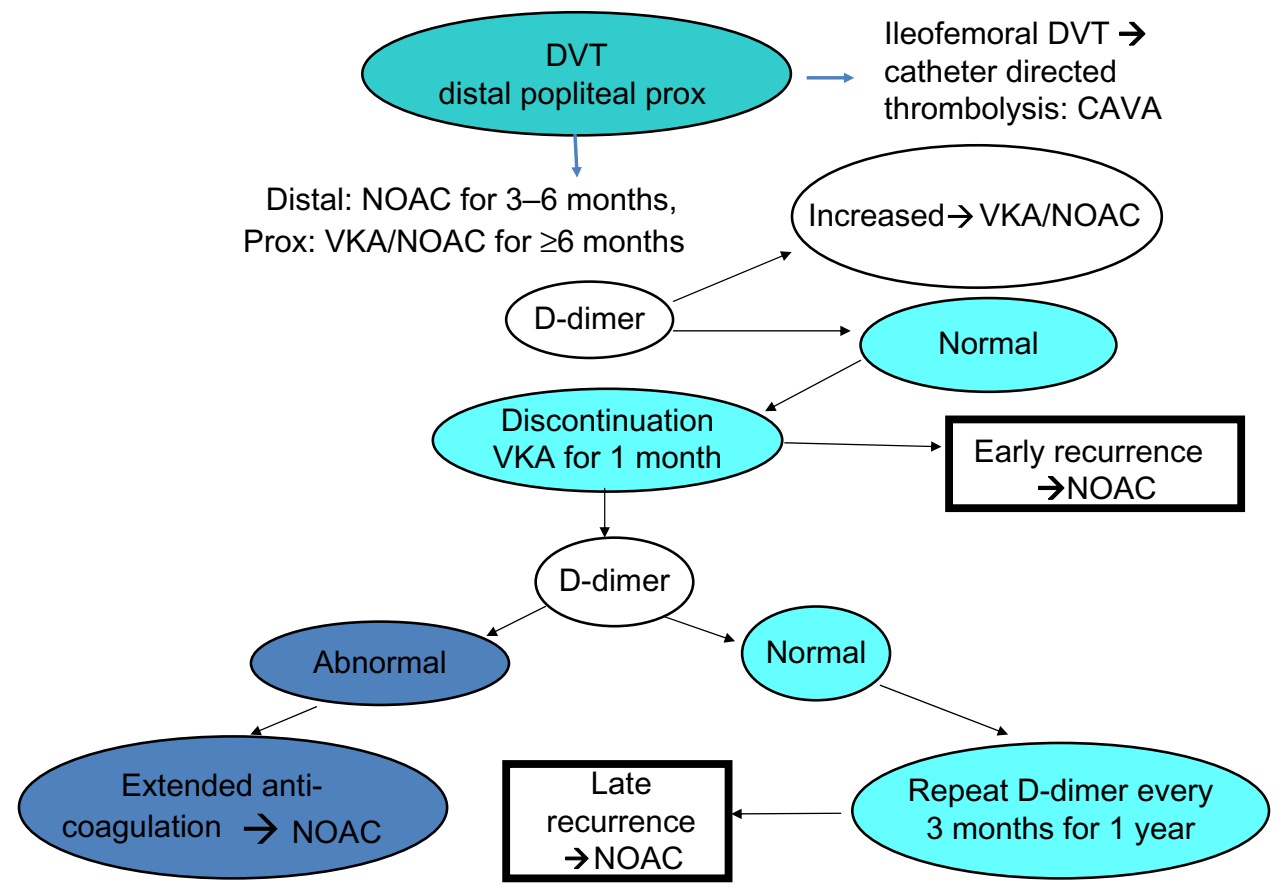

Figure 7 Algorithm of the Rotterdam modification of the PROLONG study for the duration of anticoagulation and the compelling need to extend anticoagulant in postDVT patients, preferentially with low doses of the Direct Xa (DOXa) inhibitors Rivaroxaban ${ }^{\circledR} 10 \mathrm{mg}$ once daily; Apixaban ${ }^{\circledR} 2.5 \mathrm{mg}$ twice daily, similar to what is done in the orthopedic surgical setting.

Notes: This algorithm is in accordance with the objective score assessment from, which has to be evaluated in prospective management studies in the primary care setting and in outpatient wards. 11,29-34,51 Rivaroxaban (Bayer, Leverkussen, Germany); Apixaban (Pfizer, New York City, NY, USA).

Abbreviations: DVT, deep vein thrombosis; VKA, vitamin K agonist; NOAC, novel oral anticoagulants; PTS, postthrombotic syndrome.

$50 \%$ of post-DVT patients at 3-6 months post-DVT. In the Maastricht study, reflux on CUS was present in 74 of 101 (73.3\%) tested patients. ${ }^{35}$ The cumulative incidence of PTS was $13.3 \%$ at 6 months, $17.0 \%$ at 12 months, and $21.1 \%$ at 24 months. Varicosis or venous insufficiency (present in 11\% of patients at baseline) was significantly associated with PTS (hazard ratio: 3.2) range (1.2-9.1). In the Maastricht study, patients with a low probability for developing PTS could be identified at 6 months post-DVT. ${ }^{35}$ The results of the Italian $^{33,51}$ and Dutch ${ }^{49}$ DVT-PTS studies indicated that DUS assessment 1 month, 3 months, and 6 months post-DVT was mandatory on top of objective PTS assessment according to CEAP score at 6 months, 1 year, and 2 years post-DVT, on the basis of which we designed the prospective clinical safety outcome study shown in Figures 5-7.

\section{The role of MECS in the treatment of PTS}

A clinical examination of the assessment of the risk on PTS by DUS should be performed in routine clinical practice at 3 months, 6 months, 1 year, and 2 years post-DVT to determine whether there is still a need for wearing MECS, and to see whether additional treatment is necessary (Figure 5).
About half of the DVT patients who have normal DUS 3 months post-DVT do not develop PTS after 3-9 months post-DVT; they do not need to wear MECS and anticoagulation can be stopped at 6 months post-DVT (Figures 5 and 6; Table 4). ${ }^{35-42}$ If no pathologic changes remain that means complete vrecanalization, and no reflux, the venous system functions normally and no PTS exists or will develop, the MECS do not need to be worn any longer and the asymptomatic patient can be discharged from the follow-up after 6 months (normal duplex Figure 5; Table 4). In the event that reflux of the deep venous system is found at and after 6 months postDVT, ${ }^{43-46}$ wearing MECS and OAT (VKA or novel oral anticoagulants [NOAC]) should be continued (Figures 5 and 6). In the case of RVO after 3 months or 6 months post-DVT, according to extensive CDUS evaluation, additional strategies will mainly depend on the site and extension of RVO. If this involves only the femoral and/or popliteal veins and calf veins, continuation of MECS is advocated. If DUS raises suspicion only for residual obstruction at the inferior vena cava or iliofemoral level (with the absence of phasic flow at the level of the common femoral vein, visible obstruction of the external iliac vein, or prominent collateral circulation mostly crossing the pubis area), venography is performed. 
In most cases, puncture of the popliteal vein is used to access the deep venous system. Venous ulcers are treated according to modern evidence-based guidelines. ${ }^{38,39}$

\section{DVT and PTS bridging the gap in prospective studies}

In view of the proposed concept in Table 4 and Figure 5, four unanswered questions in the treatment of provoked and unprovoked DVT are:

1. Are 3 months and 6 months post-DVT the appropriate time points to determine the group of patients who do not have PTS and the group who do develop PTS? If yes, then this is to be demonstrated in a cost-effectiveness outcome study.

2. Which post-DVT patient has a clear indication for longterm compression therapy, so as to prevent PTS after the initial treatment in the acute phase of DVT? In symptomatic DVT and PTS legs to relieve symptoms with no or minor effect on DVT recurrence. To be evaluated in a prospective management study.

3. Can MECS and anticoagulation be safely discontinued at 3 months or 6 months post-DVT in the case of rapid recanalization, no reflux, and no PTS? If yes, then this is to be demonstrated in a cost-effectiveness outcome study.

4. Is the continuation of MECS and the extension of anticoagulation mandatory in the event of reflux with or without PTS at 6 months, 12 months, and 24 months post-DVT indicated for the prevention of DVT recurrence and for the increase of PTS severity? If yes, then this is to be demonstrated in a cost-effective, safety outcome study.

The diagnostic work up of post-DVT patients should follow the Rotterdam approach to PTS, according to according to Michiels et al, 2,3,7,49 for the indication of wearing stockings (Figure 5). To address these questions, we propose a prospective safety outcomes study with a follow-up period of 1-5 years (Figures 5 and 6). Patients with provoked or unprovoked DVT at the time of diagnosis were included. All DVT patients will (according to the standard) immediately receive anticoagulant and compression therapy. Objective documentation will consist of PTS score assessments and DUS, plus ambulant venous pressure measurements, in order to make a therapeutic decision at time points 1 month, 3 months, 6 months, 9 months, 12 months, and 24 months post-DVT (Figures 5 and 6). Based on these objective measurements and assessments of PTS, DVT patients will be risk stratified at 6 months post-DVT for the continuation or discontinuation of compression therapy with MECS and anticoagulation, according to the study design (Figures 5 and 6), followed by discontinuation when no evidence of reflux obstruction or PTS symptoms are present.

Complete recanalization within 1-3 months and no reflux is predicted to be associated with no PTS, obviating the need for anticoagulation and MECS at 6 months post-DVT according to Michiels et $\mathrm{al}^{47-49}$ (Figures 6 and 7). On the other hand, delayed incomplete or complete recanalization at 6-9 months post-DVT is usually complicated by reflux due to valve destruction, thereby indicating the need to continue anticoagulant treatment and the need to wear MECS (Figure 6). Post-DVT patients with no PTS and rapid recanalization within 3 months, no reflux, and no PTS at 6 months postDVT do not need to wear MECS, and anticoagulation can be discontinued at 6 months post-DVT. ${ }^{47,48}$ This strategy from study arm 1 is predicted to be cost effective and safe. Patients with delayed recanalization and reflux with or without RVT, but with no significant PTS (CEAP 1-3) 3-9 months postDVT, while wearing MECS, are candidates for extended anticoagulation for at least 1 year or 2 years, and they should be reevaluated 1 year post-DVT (Figures 5 and 6). ${ }^{4-49}$ Symptomatic patients with PTS (identified by an increased Villalta score or CEAP 3-4) 6 months post-DVT are candidates for wearing stockings for symptoms relief at work and when walking in the daytime. Patients with PTS, according to both the subjective Villalta and objective CEAP scores in addition to DUS at 6-12 months post-DVT, should be treated with oral anticoagulants for 2 years, and they should be considered for extended or even lifelong anticoagulation with VKA or NOAC in combination with MECS (Figure 7). As demonstrated by Palareti et $\mathrm{al}^{33}$ in the PROLONG study, proper extension of anticoagulation with VKA or NOAC is key for the prevention of DVT recurrence, as it is the best option to prevent DVT recurrence and reduce the severity of PTS. Michiels et $\mathrm{al}^{49}$ have already started a pilot study to test the feasibility of planning a multicenter prospective management and safety outcome study, according to the proposed concept shown in Figure 6, in the primary care setting. PTS is a chronic condition that affects the deep venous system, and it may extend to the superficial venous system of the legs in patients with a documented history of DVT, as discussed in our clinical review on bridging the gap between DVT and the PTS by Michiels et al. ${ }^{49}$

According to Iorio et $\mathrm{al},{ }^{50}$ in 2010 , the acceptable expected rate of recurrent VTE after the discontinuation of anticoagulation in post-DVT patients with a first unprovoked DVT was $<4 \%$ in the first year and $<6.7 \%$ after 2 years with the 
discontinuation of OAT and VKA. As an extension of the PROLONG study, Cosmi et $\mathrm{al}^{51}$ performed the DULCIS (D-dimer and ultrasound in combination Italian study) to establish the optimal duration of anticoagulation for VTE in 988 evaluable DVT patients with a first unprovoked DVT. After at least 3 months of anticoagulation, D-dimer levels were measured and DUS was performed to measure RVT $($ RVT $<4$ $\mathrm{mm}$ ), and followed according to two main strategies. Firstly, if the D-dimer level was below the age- and sex-specific cut-off values for each of the different D-dimer assays used, then anticoagulation was stopped and the D-dimer levels were reassessed at 15 days, 30 days, 60 days, and 90 days. ${ }^{51}$ If at the period of at least 6 months post-DVT the D-dimer level remained below the cut-off point, anticoagulation was definitely stopped and patients were followed-up for 2 years. In the cohort of 109 post-DVT patients who refused OAT treatment, and who had at least one D-dimer measurement above the cut-off value, the incidence of major VTE was $8.8 \%$, and distal DVT or SVT was $2.3 \%$ patient/years. In 506 $(51 \%)$ of the 988 analyzed patients, all D-dimer levels were below the cut-off point at 3 months (90 days) after stopping anticoagulation. The incidence of VTE was $2.8 \%$, distal DVT was $1.1 \%$, and SVT was $2.3 \%$ patient/years. ${ }^{51}$ Secondly, if one of the D-dimer levels was above the cut-off value 3 months (90 days) after discontinuation, then anticoagulation was resumed. This cohort of 373 patients with increased D-dimer levels that fell above the age- and sex-adjusted cut-off levels received OAT for 2 years follow-up and only four VTE events $(0.7 \%$ patient/years) were observed at the cost of 14 major bleedings $\left(2.3 \%\right.$ patients/years). ${ }^{51}$ These data may indicate that the upper limit of a normal D-dimer test in post-DVT patients with early PTS does not seem to be low enough for safe DVT recurrence prevention. The presence of HER signs in PTS in either leg (CEAP 3 or CEAP 4) a lower ELISA VIDAS D-dimer cut-off level of $250 \mathrm{ng} / \mathrm{mL}$ is recommended in unprovoked DVT for lowering the risk on DVT recurrence after anticogulation discontinuation in post-DVT patients at 6 months post-DVT. ${ }^{33,52}$

In the proposed prospective study design that aims to bridge the gap between DVT and PTS, in patients with a first DVT provoked by a transient reversible factor (for example, surgery or a recent trauma), or in those with a provoked DVT who have a low risk of recurrent VTE 3-6 months post-DVT, it was determined that OAT can safely be discontinued (study arm 1; Figures 5 and 6). The decision to extend and/or to continue OAT indefinitely after completing 6 months of anticoagulant therapy for proximal DVT in unprovoked DVT is one of the most important unanswered questions in VTE management in the REVERSE studies. ${ }^{11-13,52}$ The present critical appraisal of the literature is in line with the suggestion that the early signs of PTS, with persistent abnormalities on DUS at 6 months post-DVT, and elevated D-dimer levels after the discontinuation of OAT are the main independent predictors of DVT recurrence and embolic complications (VTE). The combined use of each of these important univariate or single predictors for recurrent VTE will be sufficient in segregating patients at 6 months post-DVT into low enough $(<3 \%$ person/year) groups to provide clinicians and patients with the comfort to confidently discontinue OAT.

A complete and detailed work-up and follow-up - as proposed in Figures 6 and 7, which expand upon the results of the PROLONG and DULCIS studies ${ }^{33,51}$ - are a significant step ahead to bridging the gap between DVT and PTS. To realize such a prestigious project, a multidisciplinary team of primary care physicians, clinicians, phlebologists, vascular internists, and radiologists is mandatory when cooperating in conducting transparent prospective management studies, to improve risk stratification of provoked and unprovoked DVT patients at 6 months post-DVT, for the indication to extend anticoagulation for 2 years, and so welldefined PTS patients can be followed-up for several years (or even throughout their lives). The Canadian VTE Study Group ${ }^{18,19,52}$ conducted a multicenter, prospective, cohort, clinical decision rule derivation of 646 patients with a first unprovoked VTE treated with OAT for about 6 months, and the patients were followed for recurrent VTE after OAT discontinuation. The PTS signs of HER (CEAP $\geq 3$ ) appeared to make biological and clinical sense as important risk factors for DVT recurrence and embolic complications. The presence of HER, as assessed by subjective Villalta by clinicians and objective CEAP criteria by phlebologists, on top of CUS abnormalities by phlebologists or radiologists at time points 3, 6 to 12 months post-DVT (Figures 6 and 7), surely will be the strongest predictors of DVT recurrence and embolic complications.

In view of the incidence and distribution of acute DVT in the propsed study design in Figures 5, 6, and 7 will have significant therapeutic implications. Symptomatic patients with acute iliofemoral DVT and rapid extension of proximal DVT into the ileofemoral region are candidates for catheterdirected thrombolysis in the hands of DVT experts, as the risk of severe PTS by anticoagulation alone is irreversible and high. Recanalization of distal DVT in the calf and lower popliteal region is predicted to be rapid and complete, with no reflux on DUS and no PTS according to the CEAP classification, obviating the need to wear stockings 
and receive anticoagulation therapy at 6 months post-DVT. Delayed and incomplete recanalization in proximal DVT, as well as the presence of reflux due to valve destruction (irrespective of the degree of recanalization on DUS at 3-6 months post-DVT), is associated with a high risk of DVT recurrence and the development of symptomatic PTS. This indicates the need to wear MECS for the relief of symptoms, and to extend anticoagulation to prevent DVT recurrence as the main cause of PTS, preferentially with a low dose of NOAC.

\section{Acknowledgments}

The present report on the DVT-PTS Bridging the Gap study design was initiated and written by Dr Michiels in his position of Senior Investigator, Phlebology, at the Department of Dermatology, Erasmus University Medical Center, Rotterdam, the Netherlands, in the period between January 1, 2007-December 31, 2013 in a joint venture with Dr HA Martino Neumann, Rotterdam, the Netherlands, and Dr Gualtiero Palareti, Bologna, Italy. This was an invited manuscript (Proceedings of the DVT-PTS Workshop, 2nd European Chapter Congress of the International Union of Angiology; Rome, Italy; September 29, 2013).

\section{Disclosure}

The authors report no conflicts of interest in this work.

\section{References}

1. Cardiovascular Disease Educational and Research Trust; Cyprus Cardiovascular Disease Educational and Research Trust; European Venous Forum; International Surgical Thrombosis Forum; International Union of Angiology; Union Internationale de Phlébologie. Prevention and treatment of venous thromboembolism. International Consensus Statement (guidelines according to scientific evidence). Int Angiol. 2006;25(2):101-161.

2. Michiels JJ, Gadisseur A, Van Der Planken M, et al. A critical appraisal of non-invasive diagnosis and exclusion of deep vein thrombosis and pulmonary embolism in outpatients with suspected deep vein thrombosis or pulmonary embolism: how many tests do we need? Int Angiol. 2005;24(1):27-39.

3. Michiels JJ, Gadisseur A, van der Planken M, et al. Different accuracies of rapid enzyme-linked immunosorbent, turbidimetric, and agglutination D-dimer assays for thrombosis exclusion: impact on diagnostic workups of outpatients with suspected deep vein thrombosis and pulmonary embolism. Semin Thromb Hemost. 2006;32(7):678-693.

4. Elias A, Mallard L, Elias M, et al. A single complete ultrasound investigation of the venous network for the diagnostic management of patients with a clinically suspected first episode of deep venous thrombosis of the lower limbs. Thromb Haemost. 2003;89(2):221-227.

5. Schellong SM, Schwarz T, Halbritter K, et al. Complete compression ultrasonography of the leg veins as a single test for the diagnosis of deep vein thrombosis. Thromb Haemost. 2003;89(2):228-234.

6. Stevens SM, Elliott CG, Chan KG, Egger MJ, Ahmed KM. Withholding anticoagulation after a negative result on duplex ultrasonography for suspected deep venous thrombosis. Ann Intern Med. 2004;140(12): 985-991.
7. Michiels JJ, Freyburger G, van der Graaf F, Janssen M, Oortwijn W, van Beek EJ. Strategies for the safe and effective exclusion and diagnosis of deep vein thrombosis by the sequential use of clinical score, D-dimer testing, and compression ultrasonography. Semin Thromb Hemost. 2000;26(6):657-667.

8. Cogo A, Lensing AW, Koopman MM, et al. Compression ultrasonography for diagnostic management of patients with clinically suspected deep vein thrombosis: prospective cohort study. $B M J$. 1998;316(7124):17-20.

9. Oudega R, Hoes AW, Moons KG. The Wells rule does not adequately rule out deep venous thrombosis in primary care patients. Ann Intern Med. 2005;143(2):100-107.

10. Siragusa S, Malato A, Anastasio R, et al. Residual vein thrombosis to establish duration of anticoagulation after a first episode of deep vein thrombosis: the Duration of Anticoagulation based on Compression UltraSonography (DACUS) study. Blood. 2008;112(3): 511-515.

11. Siragusa S, Malato A, Saccullo G, et al. Residual vein thrombosis for assessing duration of anticoagulation after unprovoked deep vein thrombosis of the lower limbs: the extended DACUS study. Am J Hematol. 2011;86(11):914-917.

12. Eklöf B, Rutherford RB, Bergan JJ, et al; American Venous Forum International Ad Hoc Committee for Revision of the CEAP Classification. Revision of the CEAP classification for chronic venous disorders: consensus statement. J Vasc Surg. 2004;40(6):1248-1252.

13. Prandoni P. The Diagnostic and Therapeutic Management of Deep Vein Thrombosis and its Sequelae [dissertation]. Amsterdam, the Netherlands: University of Amsterdam; 1992.

14. Pesavento R, Bernardi E, Concolato A, Dalla Valle F, Pagnan A, Prandoni P. Postthrombotic syndrome. Semin Thromb Hemost. 2006;32(7):744-751.

15. Kahn SR. The post-thrombotic syndrome: progress and pitfalls. $\mathrm{Br} J$ Haematol. 2006;134(4):357-365.

16. Segal JB, Streiff MB, Hofmann LV, Hoffman LV, Thornton K, Bass EB. Management of venous thromboembolism: a systematic review for a practice guideline. Ann Intern Med. 2007;146(3):211-222.

17. LE Gal G, Carrier M, Kovacs MJ, et al. Residual vein obstruction as a predictor for recurrent thromboembolic events after a first unprovoked episode: data from the REVERSE cohort study. J Thromb Haemost. 2011;9(6):1126-1132.

18. Rodger MA, Kahn SR, Wells PS, et al. Identifying unprovoked thromboembolism patients at low risk for recurrence who can discontinue anticoagulant therapy. CMAJ. 2008;179(5):417-426.

19. Rodger M, Carrier M, Gandara E, Le Gal G. Unprovoked venous thromboembolism: Short term or indefinite anticoagulation? Balancing long-term risk and benefit. Blood Rev. 2010;24(4-5):171-178.

20. Verhovsek M, Douketis JD, Yi Q, et al. Systematic review: D-dimer to predict recurrent disease after stopping anticoagulation therapy for unprovoked venous thromboembolism. Ann Intern Med. 2008;149(7): 481-490, W94.

21. Roumen-Klappe EM, den Heijer M, Janssen MC, van der Vleuten C, Thien T, Wollersheim $\mathrm{H}$. The post-thrombotic syndrome: incidence and prognostic value of non-invasive venous examinations in a six-year follow-up study. Thromb Haemost. 2005;94(4):825-830.

22. Aschwanden M, Jeanneret C, Koller MT, Thalhammer C, Bucher HC, Jaeger KA. Effect of prolonged treatment with compression stockings to prevent post-thrombotic sequelae: a randomized controlled trial. J Vasc Surg. 2008;47(5):1015-1021.

23. Meissner MH, Manzo RA, Bergelin RO, Markel A, Strandness DE Jr. Deep venous insufficiency: the relationship between lysis and subsequent reflux. J Vasc Surg. 1993;18(4):596-605; discussion 606-608.

24. Markel A, Manzo RA, Bergelin RO, Strandness DE Jr. Valvular reflux after deep vein thrombosis: incidence and time of occurrence. $J$ Vasc Surg. 1992;15(2):377-382; discussion 383-384.

25. Brandjes DP, Büller HR, Heijboer H, et al. Randomised trial of effect of compression stockings in patients with symptomatic proximal-vein thrombosis. Lancet. 1997;349(9054):759-762. 
26. Prandoni P, Lensing AW, Prins MH, et al. Below-knee elastic compression stockings to prevent the post-thrombotic syndrome: a randomized, controlled trial. Ann Intern Med. 2004;141(4):249-256.

27. Labropoulos N, Waggoner T, Sammis W, Samali S, Pappas PJ. The effect of venous thrombus location and the extent on the development of post-thrombotic signs and symptoms. J Vasc Surg. 2008;48(2): 407-412.

28. Kahn SR, Shapiro S, Wells PS, et al; SOX trial investigators. Compression stockings to prevent post-thrombotic syndrome: a randomised placebo-controlled trial. Lancet. 2014;383(9920:880-888.

29. Prandoni P, Lensing AW, Prins MH, et al. Residual venous thrombosis as a predictive factor of recurrent venous thromboembolism. Ann Intern Med. 2002;137(12):955-960.

30. Palareti G, Legnani C, Cosmi B, Guazzaloca G, Pancani C, Coccheri S. Risk of venous thromboembolism recurrence: high negative predictive value of D-dimer performed after oral anticoagulation is stopped. Thromb Haemost. 2002;87(1):7-12.

31. Palareti G, Legnani C, Cosmi B, et al. Predictive value of D-dimer test for recurrent venous thromboembolism after anticoagulation withdrawal in subjects with a previous idiopathic event and in carriers of congenital thrombophilia. Circulation. 2003;108(3):313-318.

32. Eichinger S, Minar E, Bialonczyk C, et al. D-dimer levels and risk of recurrent venous thromboembolism. JAMA. 2003;290(8):1071-1074.

33. Palareti G, Cosmi B, Legnani C. D-dimer testing to determine the duration of anticoagulant therapy. Curr Opin Pulm Med. 2007;13(5):393-397.

34. Latella J, Desmarais S, Miron MJ, et al. Relation between D-dimer level, venous valvular reflux and the development of post-thrombotic syndrome after deep vein thrombosis. J Thromb Haemost. 2010;8(10): 2169-2175.

35. Ten Cate-Hoek AJ, Ten Cate H, Tordoir J, Hamulyák K, Prins MH. Individually tailored duration of elastic compression therapy in relation to incidence of the postthrombotic syndrome. J Vasc Surg. 2010;52(1):132-138.

36. Kolbach DN, Sandbrink MW, Neumann HA, Prins MH. Compression therapy for treating stage I and II (Widmer) post-thrombotic syndrome. Cochrane Database Syst Rev. 2003;(4):CD004177.

37. Kolbach DN, Sandbrink MW, Hamulyak K, Neumann HA, Prins MH. Non-pharmaceutical measures for prevention of post-thrombotic syndrome. Cochrane Database Syst Rev. 2004;(1):CD004174.

38. Neumann HA. Compression therapy with medical elastic stockings for venous disease. Dermatol Surg. 1998;24(7):765-770.

39. Partsch H, Flour M, Smith PC; International Compression Club. Indications for compression therapy in venous and lymphatic disease consensus based on experimental data and scientific evidence. Under the auspices of the IUP. Int Angiol. 2008;27(3):193-219.

40. van der Wegen-Franken K, Roest W, Tank B, Neumann M. Calculating the pressure and the stiffness in three different categories of class II medical elastic compression stockings. Dermatol Surg. 2006;32(2):216-223.
41. Stain M, Schönauer V, Minar E, et al. The post-thrombotic syndrome: risk factors and impact on the course of thrombotic disease. J Thromb Haemost. 2005;3(12):2671-2676.

42. Johnson BF, Manzo RA, Bergelin RO, Strandness DE. Relationship between changes in the deep venous system and the development of the postthrombotic syndrome after an acute episode of lower limb deep vein thrombosis: a one-to-six-year follow-up. J Vasc Surg. 1995;21(2):307-312; discussion 313.

43. Labropoulos N, Leon M, Nicolaides AN, et al. Venous reflux in patients with previous deep venous thrombosis: correlation with ulceration and other symptoms. J Vasc Surg. 1994;20(1):20-26.

44. Haenen JH, Janssen MC, Wollersheim H, et al. The development of postthrombotic syndrome in relationship to venous reflux and calf muscle pump dysfunction at 2 years after the onset of deep venous thrombosis. J Vasc Surg. 2002;35(6):1184-1189.

45. van Bemmelen PS, Bedford G, Beach K, Strandness DE. Quantitative segmental evaluation of venous valvular reflux with duplex ultrasound scanning. J Vasc Surg. 1989;10(4):425-431.

46. Labropoulos N, Tiongson J, Pryor L, et al. Definition of venous reflux in lower -extremity veins. J Vasc Surg. 2003;38(4):793-798.

47. Michiels JJ, Reeder-Boertjes SWI, van den Bos RR, Wentel TD, Neumann HAM. Prospective studies on the diagnosis and management of deep vein thrombosis (DVT) and the postthrombotic syndrome filling the gap. Part 1. Deep vein thrombosid (DVT). The Rotterdam approach. Cercitari Experimentale and Medico-Chirurgicale. 2007; 2-3:86-91.

48. Michiels JJ, Reeder-Boertjes SWI, van den Bos RR, Wentel TD, Neumann HAM. Prospective studies on the diagnosis and management of deep vein thrombosis. Part 2: posthrombotic syndrome, the Rotterdam approach. Cercitari Experimentale and Medico-Chirurgicale. 2007;2-3:92-97.

49. Michiels JJ, Moosdorff W, Maasland H, et al. Duplex ultrasound, clinical score, thrombotic risk, and D-dimer testing for evidence based diagnosis and management of deep vein thrombosis and alternative diagnoses in the primary care setting and outpatient ward. Int Angiol. 2014;33(1):1-19.

50. Iorio A, Kearon C, Filippucci E, et al. Risk of recurrence after a first episode of symptomatic venous thromboembolism provoked by a transient risk factor: a systematic review. Arch Intern Med. 2010;170(19):1710-1716.

51. Palareti G, Cosmi B, Legnani C, et al. D-dimer to guide the duration of anticoagulation in patients with venous thromboembolism: a management study. Blood. 2014;124(2):196-203.

52. Rodger M, Kovacs M, Le Gal G, Anderson D, Righini M, Beaudoin T; the Reverse Investigators. The REVERSE I and II studies: impact or using HERDOO2 clinical decision rule to guide anticoagulant therapy in patients with first unprovoked venous thromboembolism. International Society on Thrombosis (ISTH). 2013;Suppl 2:oral communication 12.3.
Journal of Vascular Diagnostics and Interventions

\section{Publish your work in this journal}

The Journal of Vascular Diagnostics and Interventions is an international, peer-reviewed journal of diagnostics, focusing on non invasive vascular investigation methods involved in the evaluation of vascular diseases. The journal is committed to the rapid publication in the fields of vascular diseases. Original research, review, case reports, expert

\section{Dovepress}

opinion and commentaries are all considered for publication. The manuscript management system is completely online and includes a very quick and fair peer-review system, which is all easy to use. Visit http://www.dovepress.com/testimonials.php to read real quotes from published authors. 